\title{
Active Control of Oscillation Patterns in the Presence of Multiarmed Pitchfork Structure of the Critical Manifold of Singularly Perturbed System
}

\author{
Robert Vrabel, Marcel Abas, Michal Kopcek, and Michal Kebisek \\ Faculty of Materials Science and Technology, Slovak University of Technology in Bratislava, Hajdoczyho 1, 91701 Trnava, Slovakia \\ Correspondence should be addressed to Robert Vrabel; robert.vrabel@stuba.sk
}

Received 2 September 2013; Revised 22 September 2013; Accepted 23 September 2013

Academic Editor: Hamid Reza Karimi

Copyright (c) 2013 Robert Vrabel et al. This is an open access article distributed under the Creative Commons Attribution License, which permits unrestricted use, distribution, and reproduction in any medium, provided the original work is properly cited.

\begin{abstract}
We analyze the possibility of control of oscillation patterns for nonlinear dynamical systems without the excitation of oscillatory inputs. We propose a general method for the partition of the space of initial states to the areas allowing active control of the stable steady-state oscillations. Furthermore, we show that the frequency of oscillations can be controlled by an appropriately positioned parameter in the mathematical model. This paper extends the knowledge of the nature of the oscillations with emphasis on its consequences for active control. The results of the analysis are numerically verified and provide the feedback for further design of oscillator circuits.
\end{abstract}

\section{Introduction}

Control of oscillations is a practically important problem in many technical applications (see e.g. $[1,2]$ ), which can generally be stated in terms of two different objectives.

(a) To obtain an asymptotically stable zero solution which attracts all initial conditions in a suitably large region (regulator problem [3]).

(b) To obtain an asymptotically stable periodic solution with desired properties (such as oscillation at the given amplitudes and frequencies) and which attracts all initial states in a suitably large region (oscillator problem).

In this context, usually two-well potential of an unperturbed system was considered by using analytic methods and numerical simulations, (see, e.g., [4-6] and the references therein).

Also, in many biological systems, some form of oscillation control is needed to track the constantly changing resonant frequency or to tune it to a specified frequency while keeping the amplitude constant [7-9].

For example, in [10], the oscillation patterns were analyzed by the symmetric Hopf bifurcation theory applying group theory. Recently, in [11], the oscillation patterns of the bifurcating periodic oscillations of three coupled Van der Pol oscillators were studied.

To our knowledge, no paper exists addressing the question of active control of oscillation patterns for the dynamical systems with potential with multiwell and multibarrier structure. For example, the two-armed pitchfork bifurcation in the presence of Hopf bifurcation in the context of double magneto convection was numerically studied in the work [12]. In the paper [13], the problem of output stability for systems under the oscillatory excitation via Lyapunov approach was analyzed.

The analysis of behavior patterns is of high importance to uncover real-time threats in the industrial systems and to plan a predictive maintenance program. Recently, Yin et al. [14] proposed two online schemes for the fault-tolerant architecture for the purpose of fault-tolerant control. One is a gradient based iterative tuning scheme for the online optimization of the system performance, and the other is an adaptive residual generator scheme for the online identification of the abnormal change of the system parameters.

There are several approaches to the topic of vibration control in industrial equipment; for example, in the work [15], the symbolic computation systems are presented with the 
purpose of analyzing the spectrum and waveform vibration allowing bearing defects in low-speed machines via dynamic modeling of patterns of behavior. The paper [16] provides a comparison study on the basic data-driven methods for process monitoring and fault diagnosis. Most recently, in the paper by Palacios-Quinonero et al. [17] by using an approach based on the connected control method, activepassive structural vibration control strategies for seismic protection of multibuilding systems are designed and applied on the proposed linear mathematical model of connected multistructure mechanical systems.

The method we propose could provide the mechanical engineers the mathematical tool for an accurate diagnosis in the vibration analysis and thus prevent or predict future failures of industrial systems. The main advantage of our approach-an analysis of the time-reverse differential equations associated with the problem under considerationover methods mentioned above lies in its straightforward extension to nonlinear models (continuous and discrete) with time-delay; for the topic, see, e.g., [18-20].

In this paper, we focus our attention on the proof of the existence and the possibility of active control of stable steady-state nonlinear oscillations in the dynamical system describing the singularly perturbed undamped oscillator with a continuous nonlinear restoring force and without the excitation of oscillatory inputs:

$$
\begin{gathered}
\epsilon^{2} y^{\prime \prime}+f(t, y)=0, \\
y(-\delta)=y_{0}, \quad y^{\prime}(-\delta)=y_{1},
\end{gathered}
$$

where

$$
f(t, y)= \begin{cases}y^{4 n+1} & \text { for } t \in[-\delta, 0] \\ y \prod_{i=1}^{2 n}\left(y^{2}-\left(\frac{\mu i h(T) t}{T}\right)^{2}\right) & \text { for } t \in[0, T] \\ y \prod_{i=1}^{2 n}\left(y^{2}-\mu^{2} i^{2} h^{2}(t)\right) & \text { for } t \in[T, \infty),\end{cases}
$$

$\left[y_{0}, y_{1}\right]$ is an initial state, $y_{\epsilon}\left(\cdot, y_{0}, y_{1}\right)$ is a direct output, $h$ is a positive continuous function on $[T, \infty], n \in N, \delta>0, T>$ 0 , and $\epsilon, 0<\epsilon \ll 1$ is a singular perturbation parameter. It is instructive for future reference to keep in mind the symmetric manifold $f(t, y)=0$ (Figure 1 ). The parameter $\mu>0$ is a constant determining the distance between pitchfork arms for $t \geq T$, and without loss of generality, we will assume that $\mu=1$.

Rewriting (1) to an equivalent system of three first order autonomous equations, we obtain

$$
\begin{gathered}
\epsilon y^{\prime}=w \\
\epsilon w^{\prime}=-f(t, y) \\
t^{\prime}=1 \\
y(-\delta)=y_{0}, \quad w(-\delta)=\epsilon y_{1} .
\end{gathered}
$$

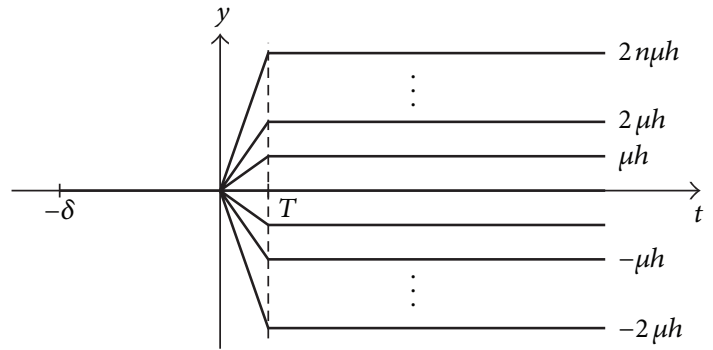

FIgURE 1: The multiarmed pitchfork manifold $f(t, y)=0$.

We show that the nonlinear system described by differential equation from (1) can basically exhibit two distinct types of steady-state oscillations, namely.

(i) In-well, small orbit dynamics, where the system state remains within the potential well centered at a stable equilibrium point (center).

(ii) Cross-well, large orbit dynamics, whose trajectories surround the $4 n+1$ equilibrium points (the saddles between centers).

The results of the analysis will be numerically verified and provide valuable insights into dynamics of the control system under consideration.

Further, we show that the singular perturbation parameter $\epsilon$ plays role modeling tool for the frequency control of the nonlinear oscillations arising in these systems. Another objective of this paper is to divide the space $\mathbb{E}^{2}$ of initial states $\left[y_{0}, y_{1}\right]$ to the areas of stability $A_{-n}(\epsilon)$, $\ldots, A_{-2}(\epsilon), A_{-1}(\epsilon), A_{0}(\epsilon), A_{1}(\epsilon), A_{2}(\epsilon), \ldots, A_{n}(\epsilon), A_{i}(\epsilon) \subset \mathbb{E}^{2}$, $i=-n, \ldots, 0, \ldots, n$ such that for $\left[y_{0}, y_{1}\right] \in A_{i}(\epsilon)$ solution of (1) oscillate around $2 i h(t), i=-n \ldots, 0, \ldots, n$ and

$y_{\epsilon}(t) \in \begin{cases}((2 i-1) h(t), \infty) & \text { for } i=n \\ ((2 i-1) h(t),(2 i+1) h(t)) & \text { for } i=-(n-1) \ldots, \\ & 0, \ldots,(n-1) \\ (-\infty,(2 i+1) h(t)) & \text { for } i=-n\end{cases}$

for $t \geq T$.

Finally, we prove that the solutions $y_{\epsilon}$ of (1) will rapidly oscillate for $t \geq T$ with the frequency of the oscillations increasing without bound as $\epsilon \rightarrow 0^{+}$.

System (3) is an example of a singularly perturbed system, because in the limit $\epsilon \rightarrow 0^{+}$, it does not reduce to a differential equation of the same type, but to an algebraicdifferential reduced system

$$
\begin{gathered}
0=w \\
0=-f(t, y) \\
t^{\prime}=1 .
\end{gathered}
$$


Another way to study the singular limit $\epsilon \rightarrow 0^{+}$is by introducing the new independent variable $\tau=t / \epsilon$ which transforms (3) to the system

$$
\begin{gathered}
\frac{\mathrm{d} y}{\mathrm{~d} \tau}=w \\
\frac{\mathrm{d} w}{\mathrm{~d} \tau}=-f(t, y) \\
\frac{\mathrm{d} t}{\mathrm{~d} \tau}=\epsilon .
\end{gathered}
$$

Taking the limit $\epsilon \rightarrow 0^{+}$, we obtain the so-called associated system [21]

$$
\begin{gathered}
\frac{\mathrm{d} y}{\mathrm{~d} \tau}=w \\
\frac{\mathrm{d} w}{\mathrm{~d} \tau}=-f(t, y) \\
\frac{\mathrm{d} t}{\mathrm{~d} \tau}=0 \quad \text { that is, } t=t^{*}=\text { constant}
\end{gathered}
$$

in which $t$ plays the role of a parameter.

Both scalings agree on the level of phase space structure when $\epsilon \neq 0$ but offer very different perspectives since they differ significantly in the limit when $\epsilon=0$. The main goal of singular perturbation theory is to use these limits to understand structure in the full system when $\epsilon \neq 0$.

The critical manifold $S$ is defined as a solution of the reduced system; that is,

$$
S:=\{(t, y, w): t \in\langle-\delta, \infty), f(t, y)=0, w=0\},
$$

which corresponds to a set of equilibria for the associated system (8)-(10).

\section{Multiarmed Pitchfork Bifurcation of Associated System at $t^{*}=0$}

Without loss of generality, we will assume hereafter that $n=1$.

Then, the equations

$$
f(t, y)=0, \quad w=0
$$

have one solution for $y, w$ if $t^{*} \in[-\delta, 0]$ and five if $t^{*} \in$ $(0, \infty)$; that is, the critical manifold is a multiarmed pitchfork manifold. Due to the fact that the eigenvalues of the Jacobian are

$$
\lambda\left(t^{*}, y, w\right)= \pm \sqrt{-\frac{\partial f}{\partial y}\left(t^{*}, y\right)},
$$

the associated system (8), (9), and $t=t^{*}=$ const has, for $n=1$, one equilibrium (degenerate center with a double zero eigenvalue) for $t^{*} \in(-\delta, 0)$ and five equilibria (two saddles and three centers) for $t^{*} \in(0, \infty)$. Thus, the two pieces of critical manifold $S$ corresponding to the saddles of associated system (8)-(10) are the normally hyperbolic submanifolds [21].

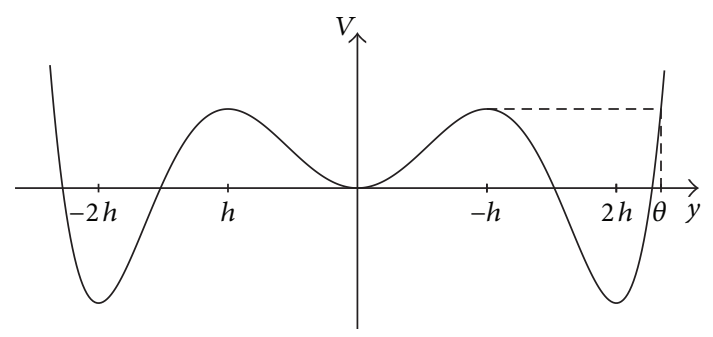

Figure 2: Potential profile $V$ with $h(t) \equiv$ const, $n=1$, and $\mu=1$ for $t \geq T . V(t, y)=V(y)$.

Consider the total energy functional

$$
\begin{gathered}
H(t, y, w)=\frac{1}{2} w^{2}+V(t, y), \\
V(t, y)=\int_{0}^{y} f(t, s) \mathrm{d} s
\end{gathered}
$$

with potential $V(t, y)$ with multibarrier and multiwell structure (for $n=1$, see Figure 2).

We use the level surfaces $H(t, y, w)=H(t)$ of $H$ on $[-\delta, \infty)$ to characterize the trajectories of (3). These surfaces in $(t, y, w)$-space are defined by

$$
w= \pm(2(H(t)-V(t, y)))^{1 / 2},
$$

extending it as long as $w$ remains real. In our case, such trajectories, lying on the surface $w=w(t, y, \epsilon)$, are bounded for every small $\epsilon$. On the interval $[-\delta, 0]$, there is a motion in a single potential well and on the interval $[0, \infty)$, triple well with a barrier in between.

Computing the derivative of $H$ along the solution of (3) ( $n=1$, for $n>1$, we proceed analogously), we obtain for $t \geq T$

$$
\begin{aligned}
H^{\prime}(t)= & w_{\epsilon}(t) w_{\epsilon}^{\prime}(t)+f\left(t, y_{\epsilon}(t)\right) y_{\epsilon}^{\prime}(t) \\
& +\int_{0}^{y_{\epsilon}(t)} \frac{\partial f}{\partial t}(t, s) \mathrm{d} s \\
= & 8 h(t) h^{\prime}(t) y_{\epsilon}^{2}(t)\left(h^{2}(t)-\frac{5}{16} y_{\epsilon}^{2}(t)\right) .
\end{aligned}
$$

For potential profile $(t \geq T)$, we have

$$
\begin{gathered}
\frac{\partial V}{\partial t} V(t, 0)=0, \\
\frac{\partial V}{\partial t} V(t, \pm 2 h(t))=-8 h^{\prime}(t) h^{5}(t), \\
\frac{\partial V}{\partial t} V(t, \pm h(t))=\frac{11}{2} h^{\prime}(t) h^{5}(t) .
\end{gathered}
$$

As follows from computation above, $h(t) \equiv$ const $\neq 0$ is a sufficient condition for the existence of nonlinear steady-state oscillations (constant amplitude). In this case, $H(t)=$ const and $V(t, y) \equiv V(T, y)$ for $t \geq T$.

To characterize input-output relationships, we determine the areas $A_{-1}(\epsilon), A_{0}(\epsilon), A_{1}(\epsilon)$ of initial states $y_{0}, y_{1}$ at the 


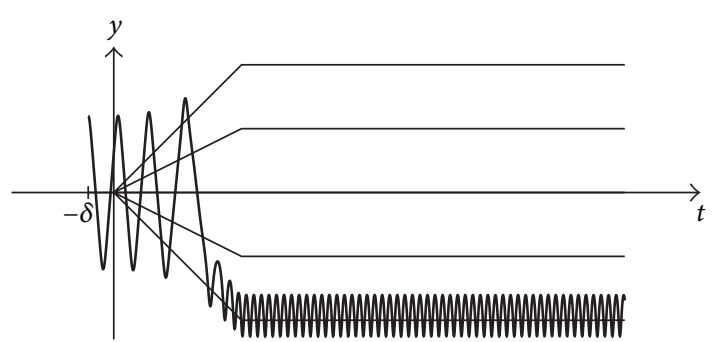

FIgURE 3: Numerical solution of $\epsilon^{2} y^{\prime \prime}+f(t, y)=0, y(-\delta)=y_{0}$, and $y^{\prime}(-\delta)=y_{1}$ with $\left[y_{0}, y_{1}\right]=[1.2,0] \in A_{-1}(\epsilon)$ and $n=1, h(t) \equiv$ $1, \mu=1, \delta=0.1, T=0.5$, and $\epsilon=0.02$.

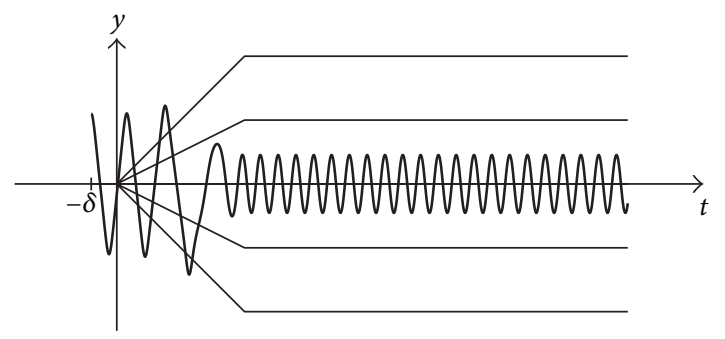

FIGURE 4: Numerical solution of $\epsilon^{2} y^{\prime \prime}+f(t, y)=0, y(-\delta)=y_{0}$, and $y^{\prime}(-\delta)=y_{1}$ with $\left[y_{0}, y_{1}\right]=[1.1,0] \in A_{0}(\epsilon)$ and $n=1, h(t) \equiv$ $1, \mu=1, \delta=0.1, T=0.5$, and $\epsilon=0.02$.

point $t=-\delta$ by solving associated time-reverse initial value problem

$$
\begin{gathered}
\epsilon^{2} \widetilde{y}^{\prime \prime}+f(T-t, \tilde{y})=0, \quad t \geq 0 \\
\tilde{y}(0)=\tilde{y}_{0}, \\
\tilde{y}^{\prime}(0)= \pm \epsilon^{-1} \sqrt{2\left(H(T)-V\left(T, \tilde{y}_{0}\right)\right)},
\end{gathered}
$$

allowing a specific switching strategy between oscillation patterns.

Let us denote $\theta_{i}$ the real roots of the six-order algebraic equation $V(T, y)=H(T)$.

Thus, taking into consideration the definition of the sets $A_{i}(\epsilon)$ and the profile of potential $V$ at $t=T$, we obtain

$$
A_{-1}(\epsilon)=\left\{\left[\tilde{y}_{\epsilon}(T+\delta),-\tilde{y}_{\epsilon}^{\prime}(T+\delta)\right]:\right.
$$

$\tilde{y}_{\epsilon}(t)$ is a solution of $(18)$,

where $H(T) \in(V(T, \pm 2 h), V(T, \pm h))$,

$$
\begin{array}{r}
\left.\tilde{y}_{0} \in\left[\theta_{1}, \theta_{2}\right], \theta_{1}<\theta_{2}<-h\right\}, \\
A_{0}(\epsilon)=\left\{\left[\tilde{y}_{\epsilon}(T+\delta),-\tilde{y}_{\epsilon}^{\prime}(T+\delta)\right]:\right.
\end{array}
$$

$\tilde{y}_{\epsilon}(t)$ is a solution of $(18)$,

where $H(T) \in(0, V(T, \pm h))$,

$$
\left.\tilde{y}_{0} \in\left[\theta_{3}, \theta_{4}\right],-h<\theta_{3}<\theta_{4}<h\right\},
$$

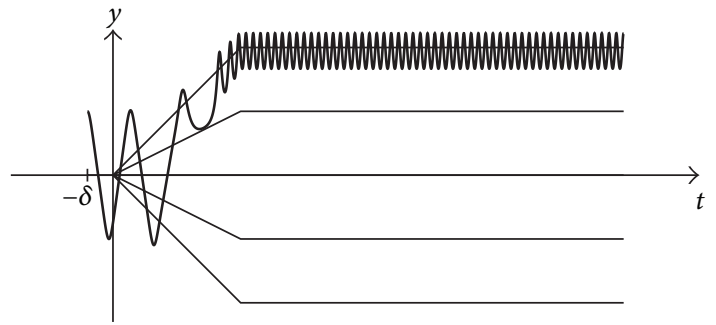

Figure 5: Numerical solution of $\epsilon^{2} y^{\prime \prime}+f(t, y)=0, y(-\delta)=y_{0}$, and $y^{\prime}(-\delta)=y_{1}$ with $\left[y_{0}, y_{1}\right]=[1,0] \in A_{1}(\epsilon)$ and $n=1, h(t) \equiv$ $1, \mu=1, \delta=0.1, T=0.5$, and $\epsilon=0.02$.

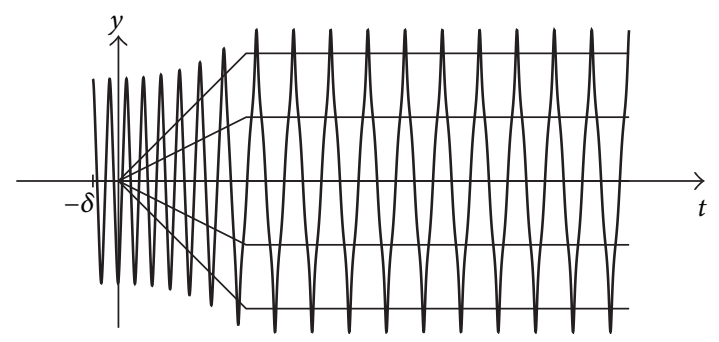

Figure 6: Numerical solution of $\epsilon^{2} y^{\prime \prime}+f(t, y)=0, y(-\delta)=y_{0}$, and $y^{\prime}(-\delta)=y_{1}$ with $\left[y_{0}, y_{1}\right]=[1.6,0] \in A_{\text {all }}(\epsilon)$ and $n=1, h(t) \equiv$ $1, \mu=1, \delta=0.1, T=0.5$, and $\epsilon=0.02$.

$$
\begin{aligned}
A_{1}(\epsilon)=\{ & {\left[\widetilde{y}_{\epsilon}(T+\delta),-\widetilde{y}_{\epsilon}^{\prime}(T+\delta)\right]: } \\
& \widetilde{y}_{\epsilon}(t) \text { is a solution of }(18), \\
& \text { where } H(T) \in(V(T, \pm 2 h), V(T, \pm h)), \\
& \left.\widetilde{y}_{0} \in\left[\theta_{5}, \theta_{6}\right], h<\theta_{5}<\theta_{6}\right\} .
\end{aligned}
$$

The Figures 3, 4, and 5 demonstrate all types of oscillations patterns for (1) with the initial values from $A_{i}(\epsilon), i=$ $-1,0,1$, respectively.

Let

$$
A_{\text {all }}(\epsilon)=\left\{\left[\tilde{y}_{\epsilon}(T+\delta),-\tilde{y}_{\epsilon}^{\prime}(T+\delta)\right]:\right.
$$$$
\tilde{y}_{\epsilon}(t) \text { is a solution of }(18) \text {, }
$$$$
\text { where } H(T) \in(V(T, \pm h), \infty) \text {, }
$$

$$
\left.\tilde{y}_{0} \in\left[\theta_{1}, \theta_{2}\right]\right\} \text {. }
$$

Then, the solutions of (1) with $\left[y_{0}, y_{1}\right] \in A_{\text {all }}(\epsilon)$ surround all pitchfork arms for $t>T$ (Figure 6). For analysis of this type of oscillations for double-well potential profile in more detail, see [22]. The selected points of the sets $A_{-1}, A_{0}, A_{1}$, and $A_{\text {all }}$ are depicted on the Figure 7.

On the basis of the Kneser and Fukuhara theory, the sets $\overline{A_{i}(\epsilon)}, i=-1,0,1$, are continua (i.e., the bounded and connected sets), and $A_{\text {all }}$ is connected in $\mathbb{E}^{2}$ where the overline denotes the closure of a set in the standard topology of $\mathbb{E}^{2}$. Obviously,

(1) $\left\{\bigcap A_{i}(\epsilon): i=-1,0,1\right.$, all $\}=\emptyset$ 


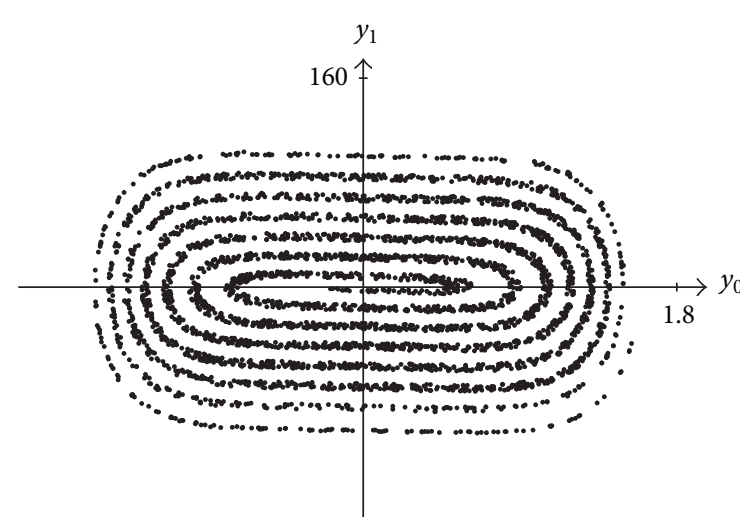

(a)

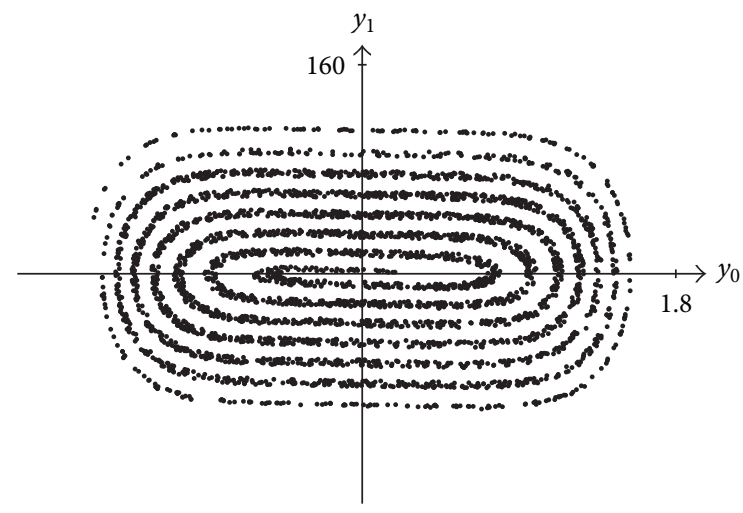

(c)

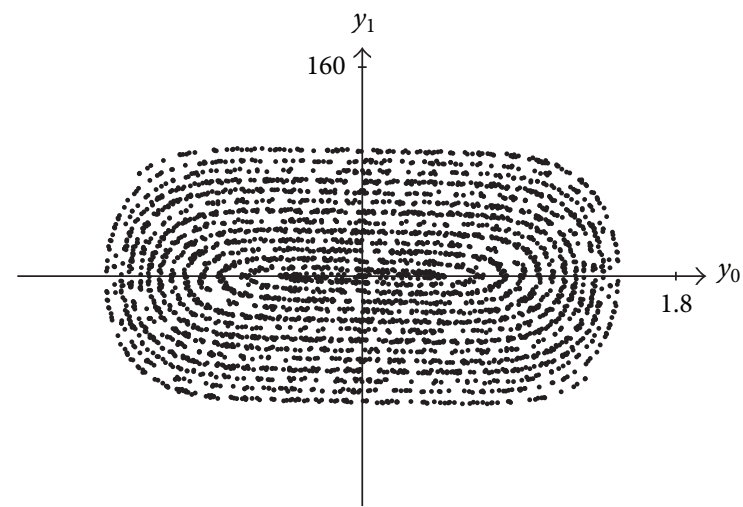

(b)

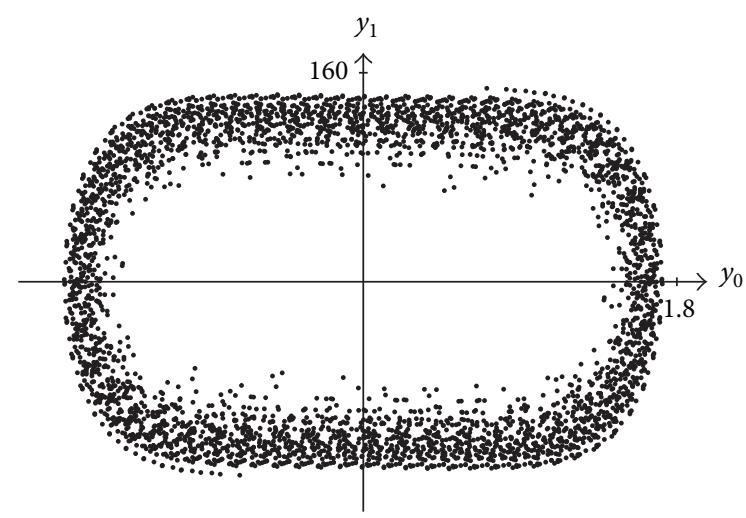

(d)

FIGURE 7: The selected points of the spiral-shaped sets $A_{-1}, A_{0}, A_{1}$, and $A_{\text {all }}$ for $n=1, h(t) \equiv 1, \mu=1, \delta=0.1, T=0.5$, and $\epsilon=0.02$ (from (a) to $(d))$

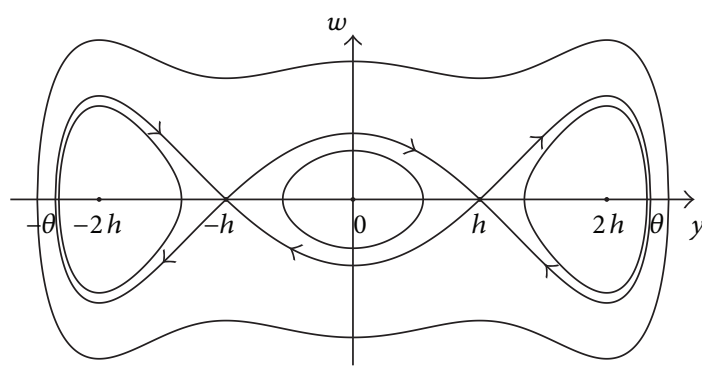

FIGURE 8: The phase portrait of system (3) for $t \geq T$ with $h(t) \equiv$ const and $n=1$. The solutions of (1) lying in the time $t=T$ on the orbits with arrows are nonoscillatory, and $\lim _{t \rightarrow \infty} y_{\epsilon}(t)=h$ or $-h$.

(2) $\left\{\bigcup \overline{A_{i}(\epsilon)}: i=-1,0,1\right.$, all $\}=\mathbb{E}^{2}$ for every $\epsilon>0$.

Also, as a consequence of the oddness of the function $f$ in the variable $y$ for every fixed $t \in[-\delta, \infty)$, we have

(1) $A_{i}(\epsilon)=-A_{-i}(\epsilon), i \neq 0$, all

(2) $A_{0}(\epsilon)$ and $A_{\text {all }}(\epsilon)$ are symmetric about the origin for every $\epsilon>0$.

\section{Nonoscillatory Solutions}

We note that there also exists for $h(t) \equiv$ const a family of the nonoscillatory solutions of (1) for $t \geq T$ with $\left[y_{0}, y_{1}\right] \epsilon$ $A_{\text {non-osc }}(\epsilon)$ where for $n=1$

$$
\begin{aligned}
& A_{\text {non-osc }}(\epsilon) \\
& =\left\{\left[\tilde{y}_{\epsilon}(T+\delta),-\tilde{y}_{\epsilon}^{\prime}(T+\delta)\right]: \widetilde{y}_{\epsilon}(t) \text { is a solution of }(18)\right. \\
& \text { with either } \\
& \text { (1) } \tilde{y}_{0}=0, H(T)=0 \text { or } \\
& \text { (2) } \tilde{y}_{0}= \pm h, H(T)=V(T, \pm h) \text { or } \\
& \text { (3) } \tilde{y}_{0}= \pm 2 h, H(T)=V(T, \pm 2 h) \text { or } \\
& \left.H(T)=V(T, \pm h), \tilde{y}_{0} \in[-\theta, \theta], \widetilde{y}_{0} \neq \pm h\right\} .
\end{aligned}
$$

In order to facilitate the understanding of the dynamics of dynamical system (3) after the time $T$, we drew the Figures 8 , $9,10,11$, and 12 . 


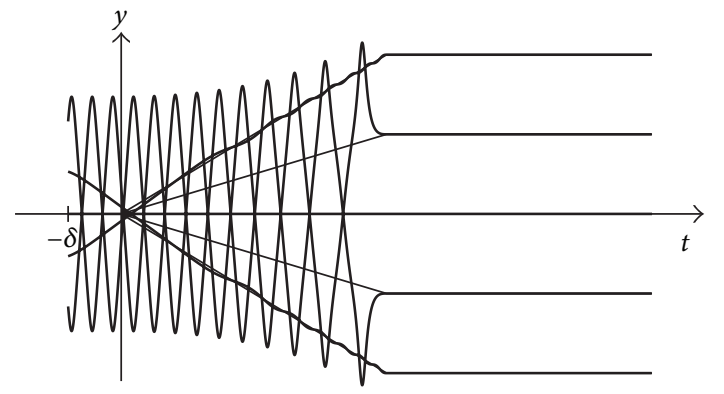

FIGURE 9: Five solutions $(n=1)$ of (1) with (1) $\tilde{y}_{0}=0$ and $H(T)=0$ $\left(y_{\epsilon}(t)=0\right.$ on $\left.[-\delta, \infty]\right),(2) \tilde{y}_{0}= \pm h$ and $H(T)=V(T, \pm h)$, and (3) $\tilde{y}_{0}= \pm 2 h$ and $H(T)=V(T, \pm 2 h)$.

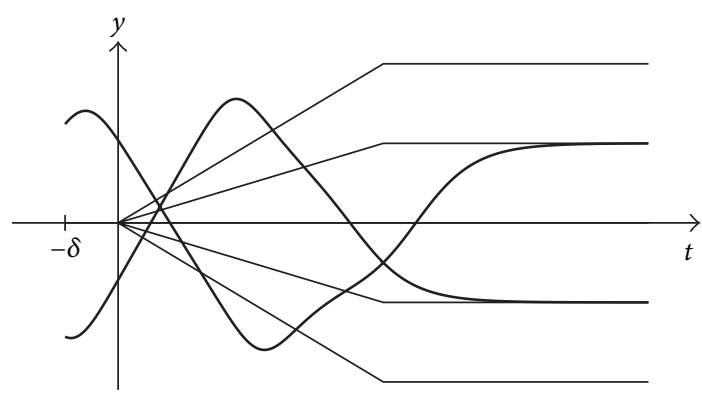

Figure 10: Two of infinitely many solutions of (1) with $\tilde{y}_{0} \in(-h, h)$ and $H(T)=V(T, \pm h)$.

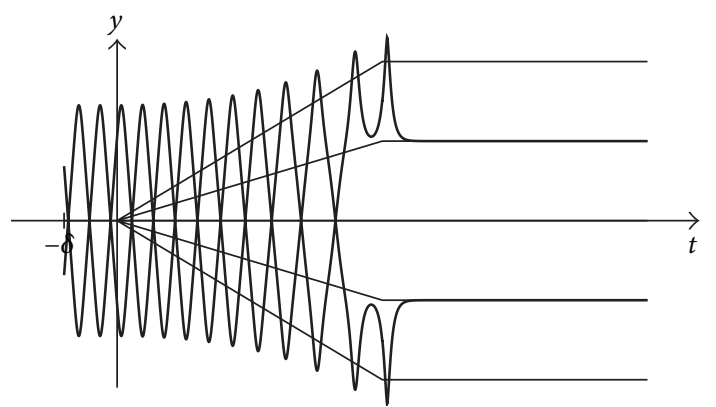

Figure 11: Two of infinitely many solutions of (1) with $H(T)=$ $V(T, \pm h)$ and either $\tilde{y}_{0} \in(h, \theta), \tilde{y}^{\prime}(0)<0$ or $\tilde{y}_{0} \in(-\theta,-h), \tilde{y}^{\prime}(0)>$ 0 .

\section{Frequency Control of Nonlinear Oscillations}

In this section, using the appropriate changes of coordinates, we show that the singular perturbation parameter $\epsilon$ plays role modeling tool for control of frequencies of nonlinear oscillations. We will analyze the case $\left[y_{0}, y_{1}\right] \in A_{1}(\epsilon)$ only. For the other cases, we proceed in a similar way.

From the theory above, it follows that the solution of (1), $\left[y_{0}, y_{1}\right] \in A_{1}(\epsilon)$ will oscillate around the half-line $y=2 h$ for $t \geq T$ (Figure 5).

Let us put $y=2 h+r(t) \cos \gamma(t)$ and $w=-r(t) \sin \gamma(t)$ for $t \geq T$. Due to requirement of steady-state oscillations,

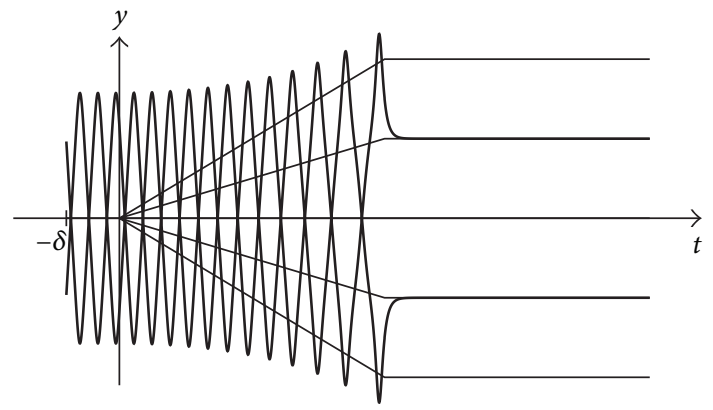

FIGURE 12: Two of infinitely many solutions of (1) with $H(T)=$ $V(T, \pm h)$ and either $\tilde{y}_{0} \in(h, \theta), \tilde{y}^{\prime}(0)>0$ or $\tilde{y}_{0} \in(-\theta,-h), \tilde{y}^{\prime}(0)<$ 0 .

$h(t) \equiv$ const, and consequently, $f(t, y)=f(y)$ for $t \geq T$. Thus,

$$
\begin{gathered}
y^{\prime}=\frac{w}{\epsilon} \Longrightarrow r^{\prime} \cos \gamma-r \gamma^{\prime} \sin \gamma=-\frac{r}{\epsilon} \sin \gamma \\
w^{\prime}=-\frac{f(y)}{\epsilon} \Longrightarrow-r^{\prime} \sin \gamma-r \gamma^{\prime} \cos \gamma=-\frac{f(y)}{\epsilon} .
\end{gathered}
$$

Multiplying these equations by $\sin \gamma, \cos \gamma$, respectively, and after algebraic manipulations, we finally obtain the following differential equation for $\gamma$ :

$$
\gamma^{\prime}=\frac{1}{\epsilon}\left[\sin ^{2} \gamma+\bar{f}(y) \cos ^{2} \gamma\right]
$$

where

$$
\begin{aligned}
\bar{f}(y) & =\frac{f(y)}{y-2 h}, \\
\bar{f}(2 h) & =\frac{\mathrm{d} f}{\mathrm{~d} y}(2 h) .
\end{aligned}
$$

Denote by $\theta$ the root of equation $V(T, y)=V(T, h)$ such that $\theta>h$ (see Figure 2) and let

$$
\begin{aligned}
& \alpha_{\Gamma}=\min \left\{\sin ^{2} \gamma+\bar{f}(y) \cos ^{2} \gamma: y \in \Gamma, \gamma \in[0,2 \pi]\right\} \\
& \beta_{\Gamma}=\max \left\{\sin ^{2} \gamma+\bar{f}(y) \cos ^{2} \gamma: y \in \Gamma, \gamma \in[0,2 \pi]\right\},
\end{aligned}
$$

where $\Gamma \subset(h, \theta)$ is a continuum depending on the constant amplitude of oscillations. Obviously, these nonzero values exist because of positivity of function $\bar{f}$ on $\Gamma$. Hence,

$$
\frac{\alpha_{\Gamma}}{\epsilon} \leq \gamma^{\prime} \leq \frac{\beta_{\Gamma}}{\epsilon}
$$

Now, let us denote by $s$ the spacing between two successive zeros of $y_{\epsilon}(t)-2 h$ on $[T, \infty)$, where $y_{\epsilon}(t)$ is a solution of (1) with $\left[y_{0}, y_{1}\right] \in A_{1}(\epsilon)$. Then, integrating the inequality (26) 
with respect to the variable $t$ between two successive zeros of $y-2 h$, we immediately obtain

$$
\begin{aligned}
& \int_{(j) \text { th zero }}^{(j+1) \text { th zero }} \frac{\alpha_{\Gamma}}{\epsilon} \mathrm{d} t \\
& \quad \leq \int_{(j) \text { th zero }}^{(j+1) \text { th zero }} \gamma^{\prime} \mathrm{d} t \\
& \quad \leq \int_{(j) \text { th zero }}^{(j+1) \text { th zero }} \frac{\beta_{\Gamma}}{\epsilon} \mathrm{d} t \\
& \frac{\alpha_{\Gamma}}{\epsilon} s \leq \pi \leq \frac{\beta_{\Gamma}}{\epsilon} s .
\end{aligned}
$$

Hence,

$$
\frac{\pi}{\beta_{\Gamma}} \epsilon \leq s \leq \frac{\pi}{\alpha_{\Gamma}} \epsilon
$$

that is, the frequency of oscillations increases without bound for $\epsilon$ tending to zero. As a consequence of the inequality (28) and the definition of the constant $\alpha_{\Gamma}$, we note that for a fixed value of parameter $\epsilon$, the greater amplitude of oscillations leads to the lower frequency of oscillations.

The data for Figures 3-12 have been worked out with the computer system MAXIMA [23, 24]. The computer code is available from the authors upon request.

\section{Conclusions}

In this paper, a novel technique to active control of the oscillation patterns and their frequencies for nonlinear dynamical systems with initial condition is proposed. The method is based on solving the time-reverse differential equation on a particular interval. The analytical results are numerically simulated by employing the computer system MAXIMA. The ideas of this paper may be naturally extended and adapted to wide class of the systems to find the basins for different patterns of systems behavior and may be employed without any principal limitation.

\section{References}

[1] A. L. Fradkov and A. Y. Pogromsky, Introduction to Control of Oscillations and Chaos, World Scientific, River Edge, NJ, USA, 1998.

[2] J. Guckenheimer and P. Holmes, Nonlinear Oscillations, Dynamical Systems, and Bifurcations of Vector Fields, Springer, New York, NY, USA, 1983.

[3] S. Park, C.-W. Tan, H. Kim, and S. K. Hong, "Oscillation control algorithms for resonant sensors with applications to vibratory gyroscopes," Sensors, vol. 9, no. 8, pp. 5952-5967, 2009.

[4] S. Lenci and G. Rega, "Optimal control of nonregular dynamics in a duffing oscillator," Nonlinear Dynamics, vol. 33, no. 1, pp. 71-86, 2003.

[5] A. H. Nayfeh and D. T. Mook, Nonlinear Oscillations, Pure and Applied Mathematics, Wiley-Interscience, New York, NY, USA, 1979.
[6] E. Tamaseviciute, A. Tamasevicius, G. Mykolaitis, S. Bumeliene, and E. Lindberg, "Analogue electrical circuit for simulation of the Duffing-Holmes equation," Nonlinear Analysis: Modelling and Control, vol. 13, no. 2, pp. 241-252, 2008.

[7] D. E. Nelson, A. E. C. Ihekwaba, M. Elliott et al., "Oscillations in NF- $\kappa \mathrm{B}$ signaling control the dynamics of gene expression," Science, vol. 306, no. 5696, pp. 704-708, 2004.

[8] T. Williamson, D. Adiamah, J. -M. Schwartz, and L. Stateva, "Exploring the genetic control of glycolytic oscillations in Saccharomyces cerevisiae," BMC Systems Biology, vol. 6, article 108, 2012.

[9] Z. Zhang, F. Xu, Z. Liu, R. Wang, and T. Wen, "MicroRNAmediated regulation in biological systems with oscillatory behavior," BioMed Research International, vol. 2013, Article ID 285063, 7 pages, 2013.

[10] A. Takamatsu, R. Tanaka, T. Yamamoto, and T. Fujii, "Control of oscillation patterns in a symmetric coupled biological oscillator system," in Proceedings of the AIP Conference, pp. 230-235, 2003.

[11] Y. Song, J. Xu, and T. Zhang, "Bifurcation, amplitude death and oscillation patterns in a system of three coupled van der Pol oscillators with diffusively delayed velocity coupling," Chaos, vol. 21, no. 2, Article ID 023111, 2011.

[12] A. M. Rucklidge, N. O. Weiss, D. P. Brownjohn, and M. R. E. Proctor, "Oscillations and secondary bifurcations in nonlinear magnetoconvection," Geophysical and Astrophysical Fluid Dynamics, vol. 68, no. 1-4, pp. 133-150, 1993.

[13] T. Hu, A. R. Teel, and Z. Lin, "Lyapunov characterization of forced oscillations," Automatica, vol. 41, no. 10, pp. 1723-1735, 2005.

[14] S. Yin, H. Luo, and S. X. Ding, "Real-Time implementation of fault-tolerant control systems with performance optimization," IEEE Transactions on Industrial Electronics, 2013.

[15] A. Tinnirello, E. Gago, and M. Dadamo, "Spectral vibration patterns by symbolic computation systems," in Proceedings of the 14th Recent Advances in Applied Mathematics Conference, Recent Advances in Applied Mathematics, pp. 263-267, 2009.

[16] S. Yin, S. X. Ding, A. Haghani, H. Hao, and P. Zhang, "A comparison study of basic data-driven fault diagnosis and process monitoring methods on the benchmark Tennessee Eastman process," Journal of Process Control, vol. 22, pp. 15671581, 2012.

[17] F. Palacios-Quinonero, J. M. Rossell, J. Rubi-Masseg, and H. R. Karimi, "Structural vibration control for a class of connected multistructure mechanical systems," Mathematical Problems in Engineering, vol. 2012, Article ID 942910, 23 pages, 2012.

[18] J. Qiu, G. Feng, and J. Yang, "A new design of delay-dependent robust $\mathrm{H}_{\infty}$ filtering for discrete-time T-S fuzzy systems with time-varying delay," IEEE Transactions on Fuzzy Systems, vol. 17, no. 5, pp. 1044-1058, 2009.

[19] J. Qiu, G. Feng, and J. Yang, "Delay-dependent nonsynchronized robust $\mathrm{H}_{\infty}$ state estimation for discrete-time piecewise linear delay systems," International Journal of Adaptive Control and Signal Processing, vol. 23, no. 12, pp. 1082-1096, 2009.

[20] Y. Wei, J. Qiu, H. R. Karimi, and M. Wang, "A new design of $\mathrm{H}_{\infty}$ filtering for continuous-time Markovian jump systems with time-varying delay and partially accessible mode information," Signal Processing, vol. 93, pp. 2392-2407, 2013.

[21] C. K. R. T. Jones, "Geometric singular perturbation theory," in C. I. M. E. Lectures, Montecatini Terme, vol. 1609 of Lecture Notes in Mathematics, Springer, Heidelberg, Germany, 1995. 
[22] R. Vrabel and M. Abas, "Frequency control of singularly perturbed forced duffing's oscillator," Journal of Dynamical and Control Systems, vol. 17, no. 3, pp. 451-467, 2011.

[23] R. H. Rand, "Introduction to Maxima," Department of Theoretical and Applied Mechanics, Cornell University, 2010, http://maxima.sourceforge.net/docs/intromax/intromax.html.

[24] W. F. Schelter, "Maxima 5.26.0 Manual," 2011, http://maxima .sourceforge.net/docs/manual/en/maxima.html. 


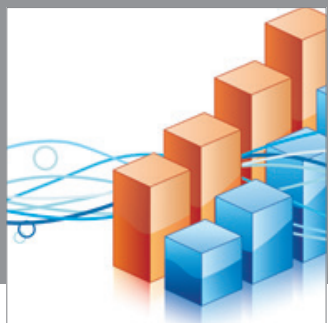

Advances in

Operations Research

mansans

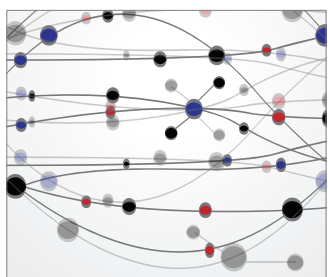

The Scientific World Journal
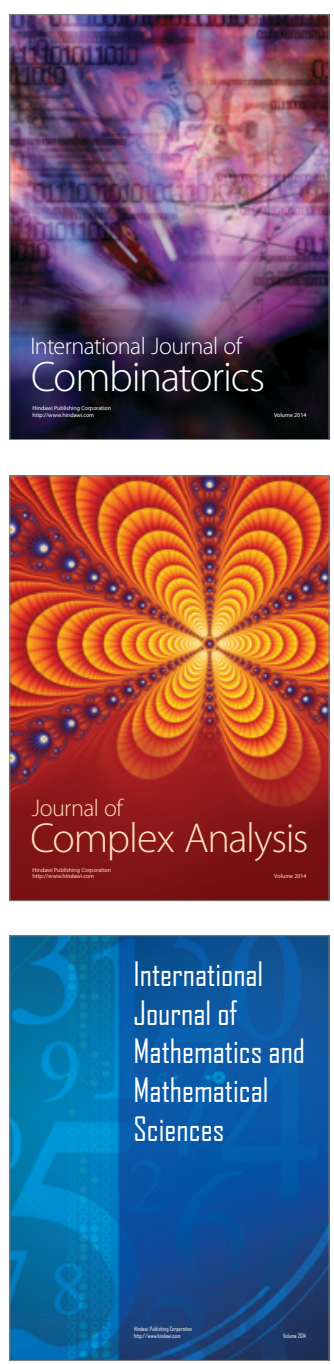
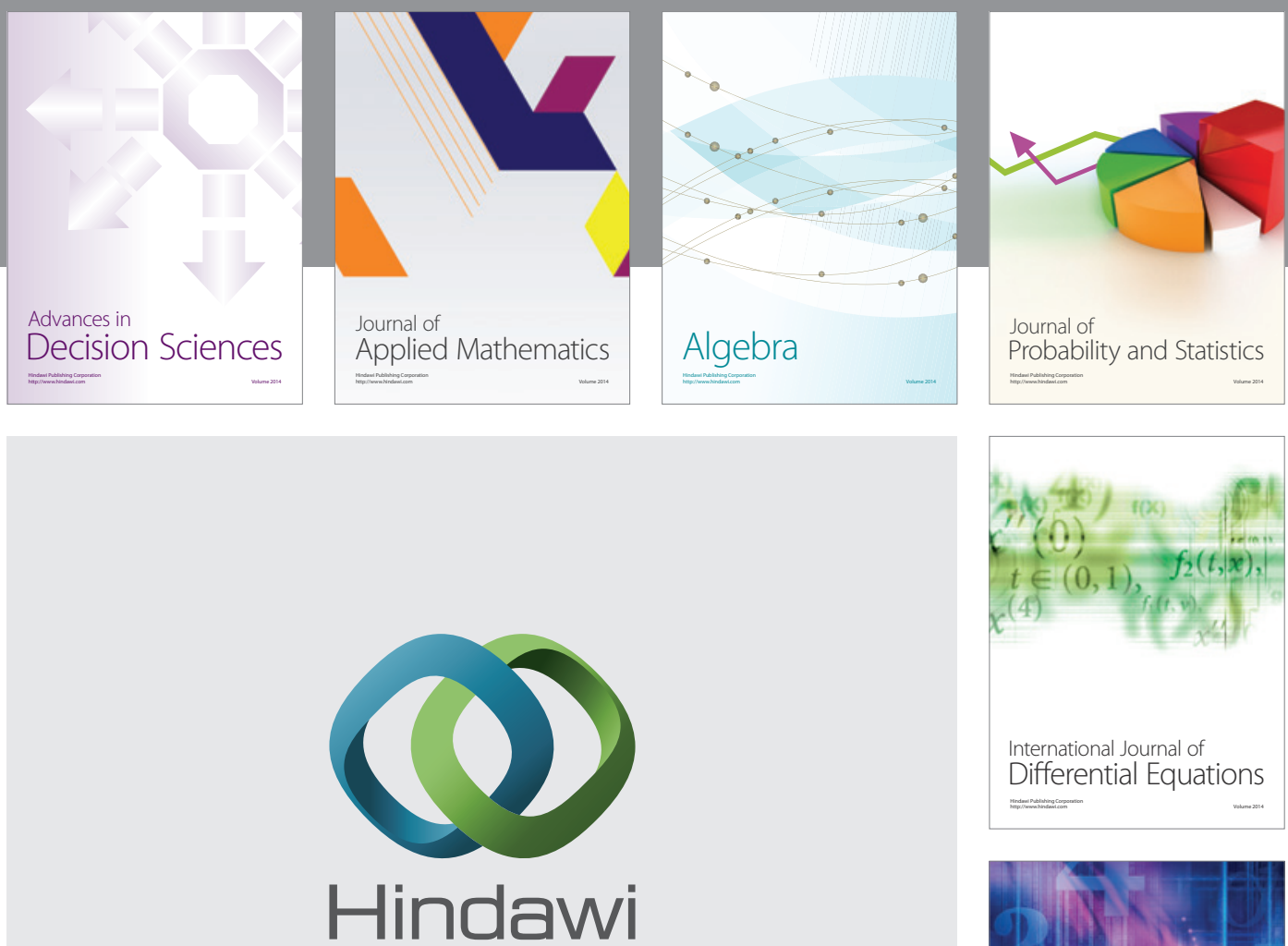

Submit your manuscripts at http://www.hindawi.com
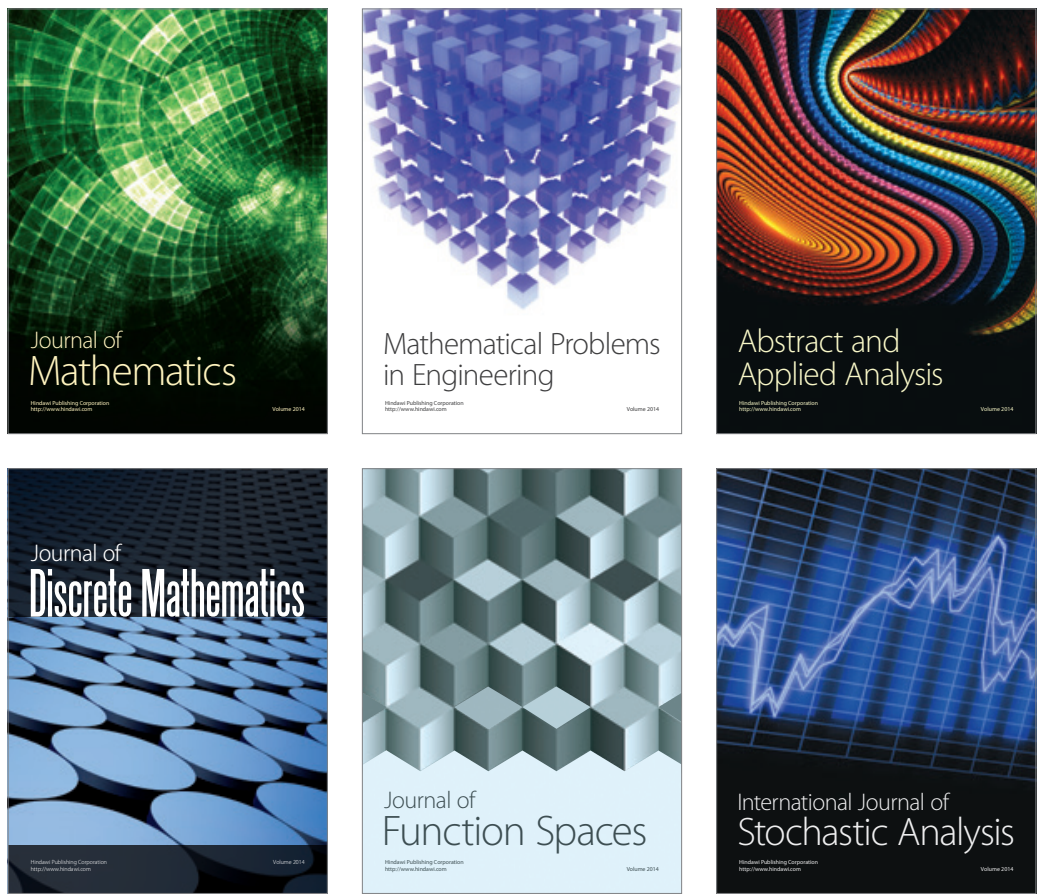

Journal of

Function Spaces

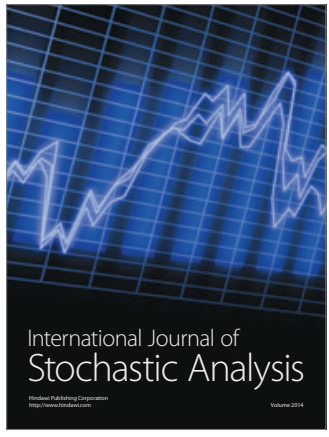

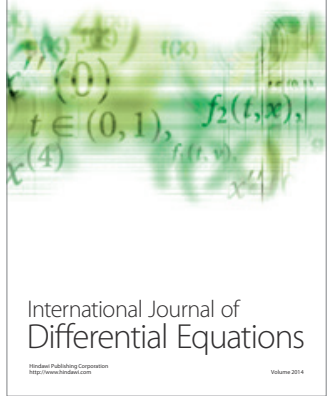
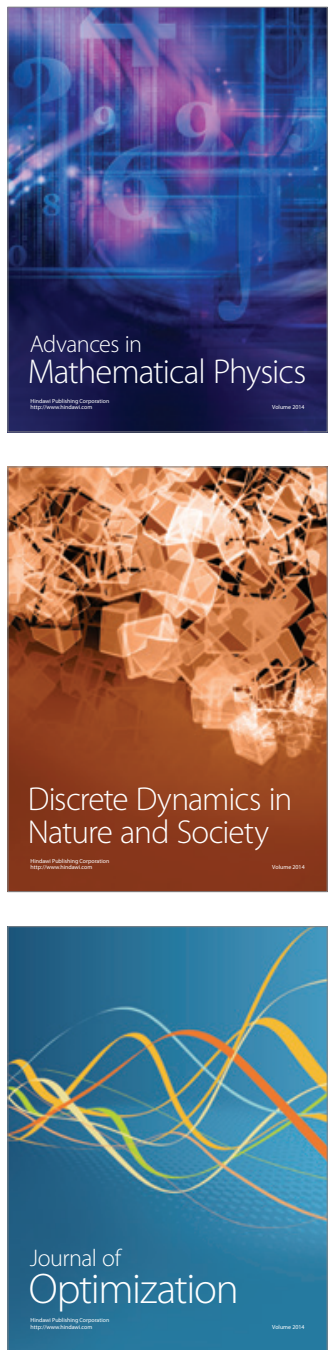